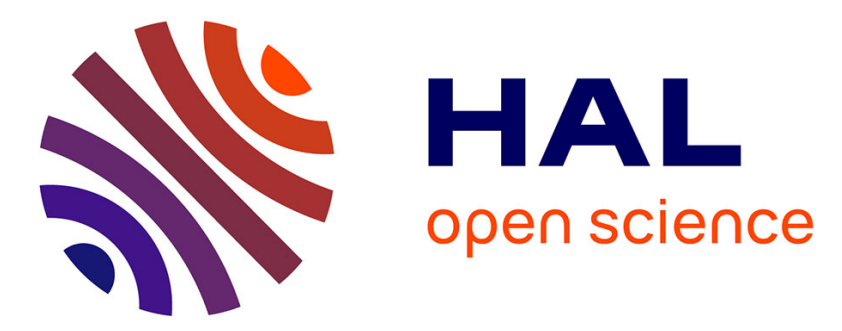

\title{
LINGO family receptors are differentially expressed in the mouse brain and form native multimeric complexes
} Anthony Guillemain, Yousra Laouarem, Laetitia Cobret, Dora Štefok, Wanyin

Chen, Solal Bloch, Amina Zahaf, Lauren Blot, Flora Reverchon, Thierry

Normand, et al.

\section{To cite this version:}

Anthony Guillemain, Yousra Laouarem, Laetitia Cobret, Dora Štefok, Wanyin Chen, et al.. LINGO family receptors are differentially expressed in the mouse brain and form native multimeric complexes. FASEB Journal, 2020, pp.1-13. 10.1096/fj.202000826R . hal-02943248

\section{HAL Id: hal-02943248 \\ https://hal.science/hal-02943248}

Submitted on 16 Nov 2020

HAL is a multi-disciplinary open access archive for the deposit and dissemination of scientific research documents, whether they are published or not. The documents may come from teaching and research institutions in France or abroad, or from public or private research centers.
L'archive ouverte pluridisciplinaire HAL, est destinée au dépôt et à la diffusion de documents scientifiques de niveau recherche, publiés ou non, émanant des établissements d'enseignement et de recherche français ou étrangers, des laboratoires publics ou privés. 


\section{LINGO FAMILY RECEPTORS ARE DIFFERENTIALLY EXPRESSED IN THE MOUSE BRAIN AND FORM NATIVE MULTIMERIC COMPLEXES}

Anthony Guillemain ${ }^{1}$, Yousra Laouarem ${ }^{2}$, Laetitia Cobret ${ }^{1}$, Dora Stefok ${ }^{1}$, Wanyin Chen ${ }^{1}$, Solal Bloch ${ }^{2}$, Amina Zahaf ${ }^{2}$, Lauren Blot ${ }^{1}$, Flora Reverchon ${ }^{1}$, Thierry Normand ${ }^{1}$, Martine Decoville $^{1}$, Catherine Grillon ${ }^{1}$, Elisabeth Traiffort ${ }^{2}$ and Séverine Morisset-Lopez ${ }^{1}$

${ }^{1}$ Centre de Biophysique Moléculaire, CNRS, UPR 4301, University of Orléans and INSERM, rue Charles Sadron 45071 ORLEANS Cedex 02, France.

${ }^{2}$ INSERM-Paris Saclay University, Diseases and Hormones of the Nervous System U1195, 80 Rue du Général Leclerc 94276 Le Kremlin-Bicêtre, France.

\section{Correspondence}

Séverine Morisset-Lopez, Centre de Biophysique Moléculaire, CNRS, UPR 4301, University of Orléans and INSERM, rue Charles Sadron 45071 ORLEANS Cedex 02, France.

Email : severine.morisset-lopez@cnrs-orleans.fr

Running title: LINGO receptors form hetero-complexes in brain 


\section{Non standard abbreviations}

BRET: bioluminescence resonance energy transfer; HA: hemagglutinin-tag; HEK 293 cells: human embryonic kidney 293 cells; HTS: high throughput screening; Ig: immunoglobulin; IP: immunoprecipitation; LAM: Lupus antigen homologous motif; LRRIG: Leucine-rich repeat and Ig domain containing protein; LRR: leucine-rich repeat; RLuc: Renilla Luciferase; RRM: RNA-recognition-motif; TBS: Tris buffer solution; TM: transmembrane domain; V2R: vasopressin 2 receptor; WB: western blot; YFP: yellow fluorescent protein. 


\begin{abstract}
Leucine-rich repeat and immunoglobin-domain containing (LRRIG) proteins that are commonly involved in protein-protein interactions play important roles in nervous system development and maintenance. LINGO-1, one of this family members, is characterized as a negative regulator of neuronal survival, axonal regeneration and oligodendrocyte precursor cell (OPC) differentiation into mature myelinating oligodendrocytes. Three LINGO-1 homologs named LINGO-2, LINGO-3 and LINGO-4 have been described. However, their relative expression and functions remain unexplored. Here, we show by in situ hybridization and quantitative polymerase chain reaction that the transcripts of LINGO homologs are differentially expressed in the central nervous system. The immunostaining of brain slices confirmed this observation and showed the co-expression of LINGO-1 with its homologs. By using BRET (bioluminescence resonance energy transfer) analysis, we demonstrate that LINGO proteins can physically interact with each of the other ones with comparable affinities and thus form oligomeric states. Furthermore, co-immunoprecipitation experiments indicate that LINGO proteins form heterocomplexes in both heterologous systems and cortical neurons. Since LINGO-1 is a promising target for the treatment of demyelinating diseases, its ability to form heteromeric complexes reveals a new level of complexity in its functioning and opens the way for new strategies to achieve diverse and nuanced LINGO-1 regulation.
\end{abstract}

Keywords: BRET/ LRRIG/ Nervous system/ Oligomerization/ Protein-protein interactions/ Transmembrane protein 


\section{INTRODUCTION}

LINGO-1 is a single transmembrane protein of 620 amino acids encoded on chromosome 15 (15q24.3) (1). It has a large extracellular region (516 amino acids) consisting in 12 leucinerich repeat motifs (LRR) and one immunoglobulin-like (Ig) domain. Three homologs of LINGO-1 exist and are called LINGO-2, LINGO-3 and LINGO-4 (2). They are structurally related to brain-enriched LRR-containing proteins, such as the Nogo 66 receptor paralogue Nogo receptor (NgR), the Netrin-G1 ligand (NGL-1), the amphoterin-induced gene and ORF (AMIGO) family, the neuronal leucine-rich repeat (NLRR) proteins and the neurotrophin tyrosine kinase receptors (RTKs) (3). LINGO-1 is selectively expressed in brain and spinal cord on both oligodendrocytes and neurons, and is not detectable in non-neural tissues $(1,4-$ 7). After a peak at postnatal day 1, LINGO-1 expression decreases in adulthood (6). Analysis of the expression pattern during early mouse development revealed that LINGO-2 and LINGO-4 mRNA were expressed only in the nervous system whereas LINGO-3 transcripts were broadly detected in many tissues across the embryo $(2,8)$. However, the neuronal functions of these homologs are still unclear in contrast to LINGO-1 whose functions have been extensively studied (9).

To explore the physiological role of LINGO-1 in vivo, various biological tools displaying the ability to block LINGO-1 function were created. They include namely LINGO-1 RNAi, dominant negative LINGO-1, a soluble form of LINGO-1 or LINGO-Fc, LINGO-1 knockout and LINGO-1 antibodies. These tools led to demonstrate three major roles for LINGO-1. The protein is a negative regulator of neuronal survival for dopaminergic, cerebellar and retinal ganglion neurons, it reduces axonal regeneration and also inhibits oligodendrocyte differentiation and myelination processes $(9,10)$. These observations as well as the ability of LINGO-1 blocking agents to promote axon regeneration, neuronal survival, and myelination rapidly suggested that the development of LINGO-1 inhibitors may be of interest for the treatment of demyelinating diseases such as multiple sclerosis (MS) (11). Therefore, the antiLINGO-1 Li81 monoclonal antibody (Opicinumab) is used in different clinical trials for such diseases. In a Phase 2 trial, this antibody showed improved latency recovery in patients with acute optic neuritis compared to placebo (11-15). However, Opicinumab did not lead to any improvement or slowdown in disability progression in MS patients. Interestingly, a more detailed analysis of the data based on magnetic resonance imaging and disease duration 
allowed to identify a subgroup of patients who may have an enhanced response to Opicinumab (16).

The molecular mechanisms involved in the regulation of these functions are mediated through the interaction of LINGO-1 with various co-factors and/or co-receptors. LINGO-1 was initially described as a co-receptor of the Nogo-A receptor $(\mathrm{NgR})$ and the p75 neurotrophin receptor $\left(\mathrm{p} 75^{\mathrm{NTR}}\right)$ or the tumor necrosis factor receptor orphan Y (TROY). The formation of these ternary complexes (LINGO-1/NgR/p75 ${ }^{\mathrm{NTR}}$ or LINGO-1/NgR/TROY) on neuronal growth cones is essential for inhibition of neurite regeneration and axonal outgrowth $(17,18)$. The ligands of these complexes, Nogo-66, myelin-associated glycoprotein (MAG) and oligodendrocyte myelin glycoprotein (OMgp), are myelin components binding to $\mathrm{NgR}$, which uses its co-receptors to transduce the activation of RhoA pathway leading to actin depolymerization and thereby to collapse or retraction of neurites (19).

LRR motifs are present in many proteins and are known to play important roles in proteinprotein interaction. Besides its interaction with the $\mathrm{NgR} / \mathrm{p} 75$ or TROY co-receptors, LINGO1 was shown to also interact with intracellular proteins or transmembrane receptors to modulate signaling pathways. LINGO-1 regulates PI3K/Akt or Ras/Mek/ERK signaling pathways through interaction with tyrosine kinase receptors including EGFR (20) and ErbB2 (21). It also interacts with the neurotrophin receptors $\operatorname{Trk} A, \operatorname{TrkB}$ and $\operatorname{TrkC}(22,23)$. The association of Trk receptors with LINGO-1 redirects Trks from the recycling pathway towards the endolysosomal pathway for degradation. Since LINGO-1 expression is upregulated after activation of the Trks receptors, LINGO-1 may function in a negative feedback loop contributing to the homeostatic regulation of neurotrophin signaling. Moreover, the LINGO-1 intracellular domain interacts with the Myt1 transcription factor (5) as well as members of serine threonine kinases including WNK1 and WNK3, which are respectively involved in LINGO-1-mediated inhibition of neurite extension (24) and neuronal apoptosis (25).

The structural data and the biophysical analysis of LINGO-1 have further shown that its extracellular part can form stable tetramers (26). We also demonstrated that LINGO-1 is able to form dimers or higher oligomers in cells expressing full-length LINGO-1 (27) indicating that the formation of oligomers is an intrinsic property that appears in the absence of its ligands or co-receptors. This oligomeric assembly could be necessary to accommodate other components of the $\mathrm{NgR}$ receptor complex to transduce the signal. In agreement with this 
hypothesis, inhibition of LINGO-1 oligomerization seems to have beneficial effects on oligodendrocyte physiology and myelination process since the monoclonal LINGO-1 antibody Li81 actually assayed in clinical trials, mediates its positive effects on oligodendrocyte maturation by blocking LINGO-1 interface required for the oligomerization of the protein (14). Moreover, small molecules affecting the conformational states of LINGO1 oligomers are also able to regulate LINGO-1 signaling (27).

In the present study, we evaluated the differential expression of LINGO homologs by realtime polymerase chain reaction, in situ hybridization and immunofluorescent staining in the mouse brain. Then, we assessed the formation of homo- and heterocomplexes of LINGO proteins in living cells using the bioluminescence resonance energy transfer (BRET) method and evaluated the physiological relevance of these interactions in brain tissues using coimmunoprecipitation experiments.

\section{METHODS}

\section{Materials}

Coelenterazine was from Interchim (Montluçon, France). The protease inhibitor cocktail was from Roche (Mannheim, Germany). PVDF membrane and CL-X film were from GE Healthcare (Chalfont St. Giles, United Kingdom). The Pierce crosslink immunoprecipitation kit Supersignal West Pico and supersignal extended Dura chemiluminescent substrates were from Thermo Fisher Scientific Inc (Rockford, Illinois, USA). All other reagents and culture media were from Sigma Aldrich (St Louis, Missouri, USA).

\section{Quantitative PCR analyses}

Brains were rapidly extracted after animal sacrifice. After dissection of the various brain regions, the tissues were rapidly frozen in liquid nitrogen and kept at $-80^{\circ} \mathrm{C}$ until use. RNA was isolated from tissues using the RNA Kit (Macherey Nagel, Düren, Germany) as recommended by the manufacturer. cDNA synthesis was performed with the Maxima first Strand Synthesis Kit (Thermo Fisher Scientific Inc) using $2.5 \mu \mathrm{g}$ of RNA from the different tissues. Quantitative polymerase chain reactions were performed on $250 \mathrm{ng}$ of cDNA with the SYBR ${ }^{\circledR}$ Premix Ex Taq ${ }^{\text {TM }}$ II (Tli RNaseH Plus) (Takara Bio Europe SAS, Saint-Germain-en- 
Laye, France). Primers to amplify LINGO homologs were mLINGO-1For, 5'GCGATTGGTGACCAAGCCGAG-3', and mLINGO-1Rev 5'GGTCCAGCAGGCGAGTCTCG-3', (for mouse LINGO-1); mLINGO-2For, 5'GAGACAAGCAAAACACAGGCCCG-3', and mLINGO-2Rev 5'GGAATGCCTTCTGGGATCGCGA-3', (for mouse LINGO-2); mLINGO-4For, 5'ACTCAGACACACGGGGAAGGTG-3', and mLINGO-4Rev 5'GGGTCTGGGAGGTGCAGTCAC-3', (for mouse LINGO-4). Mouse LINGO-3 transcripts were amplified using Qiagen® (Hilden, Germany) primers (QT00259070). PCR were conducted with LightCycler® 480 Instrument II (Roche Diagnostics, Meylan, France) and all analyses were performed with the corresponding software. The expression of hypoxanthine phosphoribosyltransferase 1 (HPRT1) gene was evaluated in all samples and used as reference to determine the relative expression of each mRNA.

\section{In situ hybridization}

In situ hybridization protocol was as previously described (28). Based on the high homology between the mouse and human homologs, the cRNA riboprobes for LINGO-1, LINGO-2 and LINGO-3 were prepared by PCR amplification of DNA fragments from vectors containing the full-length human coding sequence of each homolog (Genbank CCDS45313.1 for LINGO-1; CCDS6524.1 for LINGO-2; CCDS45905.1 for LINGO-3; CCDS30855.1 for LINGO-4). The fragments were selected for the absence of significant similarities between the different homologs and corresponded to nucleotides 890-1662 for LINGO-1, 1030-1833 for LINGO-2, 670-1643 for LINGO-3, 889-2725 for LINGO-4.

\section{Immunohistochemistry}

Mice were perfused with PBS followed by 4\% Paraformaldehyde (PFA) solution. The brain and spinal cord were collected and post-fixed in 4\% PFA before being cryo-protected in $30 \%$ sucrose in PBS. Tissues were then frozen in O.C.T (Sakura Finetek France SAS, Villeneuve d'Ascq, France). Sections (14 $\mu \mathrm{m})$ were prepared using the Cryostat Leica CM3050 S and then stored at $-20^{\circ} \mathrm{C}$. For immunostaining, frozen sections were incubated with hot citrate buffer, then blocked and permeabilized in TBS containing 1\% bovine serum albumin (BSA), $0.3 \%$ Triton and $10 \%$ foetal bovine serum (FBS) for 1 hour. The sections were then incubated 
with primary antibodies, at $4^{\circ} \mathrm{C}$ overnight in TBS containing $1 \%$ BSA, $0.3 \%$ Triton, and $10 \%$ foetal bovine serum. After extensive wash in TBS, sections were incubated with appropriate secondary antibody in TBS containing 1\% BSA and 10\% FBS, at room temperature for $1 \mathrm{~h}$ and then washing before mounting with Fluoromont-G solution (Southern Biotech, Birmingham, Alabama, USA). Each antibody was validated in the specific condition and tissue applied in the study by routine controls (omission of primary or secondary antibody). The immunofluorescence staining was visualized by fluorescent video microscope Zeiss Axiovert 200 M (Zeiss, Le Pecq, France) or confocal microscope LSM 510Meta (Zeiss) and results were analyzed by ImageJ and ZEN software.

\section{Antibodies}

The Primary antibodies used in the study are listed below, together with manufacturer, catalog number, dilutions used. The following antibodies were used: anti-hemagglutinin (HA) from Roche Diagnostics (Meylan, France); anti-LINGO-1 (AF3086, goat 1:500), anti-LINGO-1 (MAB30861, mouse 1:25) and anti-LINGO-2 (AF3679, goat 1:40) from R\&D Systems (Abingdon, United Kingdom), anti-LINGO-1 (ab23631, goat 1:250) and anti-LINGO-3 (ab169772, rabbit 1:250) from ABCAM (Cambridge, Massachusetts, USA); anti-LINGO-4 (sc-164871, goat 1:500) from Santa Cruz Biotechnology (Santa Cruz, California, USA), antiLINGO-3 (AP16177b, rabbit 1:1000) and anti- LINGO-4 (AP12795b, rabbit 1:40) from Abcepta (San Diego, California, USA), anti-GFP (Living Colors® Full-Length GFP Polyclonal Antibody, rabbit 1:2000) from BD Bioscience Clontech (San Jose, California, USA); anti-MAP2 (119942, mouse 1:500) from Sigma Aldrich. The rabbit anti-mouse (816720), goat anti-rat (629520), rabbit anti-goat (81160) and goat anti-rabbit (656120), IgG HRP-linked whole antibodies were from Life technologies (Carlsbad, California, USA). The secondary antibodies used for immunofluorescence were anti-goat DyLight594 (Ab96937, donkey 1:100), the anti-mouse TRITC (ab6786, goat 1:50), anti-rabbit A488 (Ab150077 goat, 1:1000), the sheep anti-rabbit TRITC (sheep, ab6792 1:50) were purchased from ABCAM. The goat anti-mouse A488 (A-21121, goat 1:1000) was from Invitrogen ${ }^{\mathrm{TM}}$, Life technologies.

\section{Cell culture and transfection}

HEK-293 cells were cultured in medium DMEM (Dulbecco Modified Eagle Medium) supplemented with $10 \%$ (vol/vol) foetal bovine serum, $1 \mathrm{~g} / \mathrm{L}$ glucose, $100 \mathrm{U} / \mathrm{ml}$ penicillin, 0.1 
$\mathrm{mg} / \mathrm{ml}$ streptomycin, $1 \mathrm{mM}$ glutamine. They were transfected with the calcium phosphate precipitation method as previously described (27).

\section{Western blotting}

For assessing protein expression by Western blot, cell lysates or immunoprecipitates from HEK-293 transfected cells were separated by electrophoresis on SDS/PAGE (8\% or 10\% gels) and transferred on polyvinylidene fluoride (PVDF) membranes. Blots containing HA or yellow fluorescent protein (YFP)-tagged proteins were probed with a rat anti-HA antibody (1:5000), or a rabbit anti-BD living colors full-length polyclonal antibody (1:3000). Immunoblots were also probed with goat anti-LINGO-1 (1:1000), anti-LINGO-2 (1:200), anti-LINGO-3 (1:200) and anti-LINGO-4 (1:1000) polyclonal antibodies. Horseradishperoxidase-conjugated rabbit anti-goat or anti-rat antibodies (1:33000) were used as secondary antibodies. Immunoreactive protein bands were detected using the Pico or Dura detection kit.

\section{BRET measurements}

Forty-eight hours after transfection, HEK 293 cells were detached with Versene (Thermo Fisher Scientific Inc) and resuspended in HBSS saline buffer (Thermo Fisher Scientific Inc). Intact cells or membranes were distributed in 96-well microplates (Optiplate) from PerkinElmer (Waltham, Massachusetts, USA ) and incubated for $15 \mathrm{~min}$ at $25^{\circ} \mathrm{C}$ in the absence or presence of the indicated ligands. Coelenterazine $\mathrm{H}$ substrate was added at a final concentration of $5 \mu \mathrm{M}$, and reading was performed with a Mithras LB 940 Multireader (Berthold, Bad Widbad, Germany), which allows the sequential integration of luminescence signals detected with two filter settings (Renilla Luciferase (RLuc) filter, $485 \pm 10 \mathrm{~nm}$; YFP filter, $530 \pm 12 \mathrm{~nm}$ ). Emission signals at $530 \mathrm{~nm}$ were divided by emission signals at $485 \mathrm{~nm}$. The difference between the emission ratio obtained with co-transfected RLuc and YFP fusion proteins, and the one obtained with the RLuc fusion protein alone, was defined as the BRET ratio. Results were expressed in milliBRET units (mBU, with $1 \mathrm{mBU}$ corresponding to the BRET ratio values multiplied by 1000). BRET $_{\max }$ is the maximal BRET signal obtained in milliBRET units and $\mathrm{BRET}_{50}$ represents the ratio of acceptor and donor receptors (acceptor/donor) yielding 50\% of maximum BRET signal. 


\section{Co-immunoprecipitation assays}

HEK 293 cells were co-transfected with C-terminal YFP-fused and C-terminal HA tagged proteins. For each experiment, control cells were transfected with empty plasmid controls, pcDNA3.HA. Forty-eight hours after transfection, cells were washed with ice-cold PBS and lysed in buffer containing $50 \mathrm{mM}$ Tris $\mathrm{pH} 7.5,150 \mathrm{mM} \mathrm{NaCl}, 10 \mathrm{mM}$ EDTA, $1 \%$ Triton X100 plus protease cocktail inhibitor on ice for $10 \mathrm{~min}$. The lysates were then centrifuged at 10 , $000 \mathrm{x} g$ for $10 \mathrm{~min}$. The supernatants were incubated with EZview Red anti-HA affinity gel (Sigma Aldrich) overnight at $4^{\circ} \mathrm{C}$. The beads were washed five times with lysis buffer and resuspended in 4-fold concentrated Laemmli buffer (200 mM Tris-HCl pH 6.8, 4 \% SDS, 40 $\%$ glycerol, $0.02 \%$ bromophenol, 0.5M $\beta \mathrm{ME})$.

For the analysis of heterotypic interactions in brain, the cerebral cortex was dissected from mouse brain and homogenized by sonication in lysis buffer $(50 \mathrm{mM}$ Tris- $\mathrm{HCl} \mathrm{pH} 7.5,150 \mathrm{mM}$ $\mathrm{NaCl}$, 0,27 M Sucrose, $2 \mathrm{mM}$ EDTA, 2\% SDS w/v). Samples were centrifuged at 10,000 x g for $10 \mathrm{~min}$ and the supernatants were recovered for protein concentration determination. Cortical lysate $(1000 \mu \mathrm{g})$ was then pre-clear using the control agarose Resin. In parallel the binding of $20 \mu \mathrm{g}$ LINGO-1 antibody (AF3086) to protein A/G Plus Agarose was performed by using the Pierce crosslink immunoprecipitation kit (Thermo Fisher Scientific Inc) according to the protocol provided by the manufacturer. Then, the pre-clear lysate was added to the antibodycrosslinked resin and incubated overnight at $4^{\circ} \mathrm{C}$. After washing, the beads were washed three times with lysis buffer and then eluted.

\section{Analysis of data}

All data analyses were carried out with the GraphPad Prism 4 software for Windows (GraphPad Software Inc, San Diego, CA, USA). Concentration-response curves were fitted by nonlinear regression and saturation curves by a hyperbolic one-binding site equation. The method provided estimates for $\mathrm{EC}_{50}$ values, $\mathrm{BRET}_{\max }$ and $\mathrm{BRET}_{50}$ values and corresponding SEM.

\section{RESULTS}

\section{LINGO homologs are differentially expressed in the mouse brain}

We studied the levels of LINGO mRNAs in six different adult mouse brain regions including cerebellum, hypothalamus, hippocampus, cerebral cortex, midbrain and striatum by quantitative polymerase chain reaction analysis. All LINGO mRNAs were widely expressed 
in the brain nevertheless with specific patterns for each one (Figure 1). LINGO-1 was enriched in the hippocampus and cerebral cortex. LINGO-2 transcription appeared quite homogenous throughout the different regions. LINGO-3 and LINGO-4 transcripts were more highly detected in the striatum and cerebellum, respectively. Thus, LINGO homologs are widely and differentially expressed in the adult brain.

Then, we generated riboprobes aimed at visualizing LINGO homolog mRNAs in slices of adult mouse brain by in situ hybridization. LINGO-1, LINGO-2 and LINGO-3 probes were designed in regions of the nucleotide sequences devoid of significant similarities with the other ones (Table 1) as described in Methods. Remarkably, LINGO-4 sequence did not show any similarities with its counterparts. In agreement with the above data, all four homologs could be observed in the selected regions with however a particularly low staining for LINGO-2 (Fig. 2). Moreover, we performed double in situ hybridization and immunohistofluorescence in order to determine the phenotype of the cells expressing each homolog. The majority of LINGO homolog-positive cells were neurons co-expressing the neuronal marker NeuN while less than $10 \%$ co-expressed the oligodendroglial marker Olig2 (Fig. 3 and data not shown). In a consistent manner, the neuronal marker MAP2 was widely found co-expressed with each LINGO protein in double immunohistochemistry experiments (Fig 4A and data not shown). Moreover, we observed co-localization of LINGO-1 with its homologs LINGO-2, LINGO-3 and LINGO-4 as shown in the cerebral cortex (LINGO-2, LINGO-3) or cerebellum (LINGO-4) (Fig. 4B, C and D). In a consistent manner, the neuroblastoma cell line NG-10815 expresses the four LINGO homologs (Fig S1).

\section{LINGO-1 forms constitutive hetero-oligomers with its LINGO homologs}

To address the question whether LINGO homologs can form homo- or heterocomplexes, two approaches were used to demonstrate these interactions. First, we performed coimmunoprecipitation analyses of HA-tagged and YFP-tagged LINGO proteins transiently expressed in HEK-293 cells. After immunoprecipitation of each HA-tagged protein with the anti-HA antibody, the respective YFP-tagged proteins were efficiently co-immunoprecipitated in double-transfected cells demonstrating the formation of homo-complexes indicating that as LINGO-1 (27), each homolog is able to form homodimers (Fig. 5A). The same approach was used to evaluate the ability of LINGO-1 to form heterocomplexes with its homologs and led us to show that all three YFP-tagged LINGO homologs were co-immunoprecipitated after immunoprecipitation of LINGO-1 HA (Fig. 5B). In contrast, the empty pcDNA3-HA vector 
used as a control in each co-immunoprecipitation experiment, did not lead to any signal indicating the specificity of the observed interactions.

To exclude putative artifacts generated by cell disruption and membrane solubilization, we also evaluated the physical association of LINGO homologs in intact living cells using the bioluminescence resonance energy transfer (BRET) analysis. This approach is widely used to investigate receptor interaction and more specially to study oligomerization of membrane receptors (29). BRET is strictly dependent on the molecular proximity (10-100 $)$ between the energy donor (Rluc) and acceptor (YFP), making it ideal for studying proteinprotein interactions in living cells at "physiological" expression levels. Besides allowing to avoid the disruption of the natural environment frequently altered in classical biochemical methods using detergents and membrane preparations, BRET is useful to discriminate whether the interactions occur in cis (in the same cell) or in trans (between two cells) orientation (27). For this purpose, the formation of homo- or heterocomplexes of LINGO homologs was examined in living HEK-293 cells using quantitative BRET analysis and the proper controls required by this approach $(30,31)$. After transfection of HEK-293 cells with two LINGO constructs carrying in their C-terminus end either the yellow fluorescent protein (LINGO-1-YFP; LINGO-2-YFP, LINGO-3-YFP or LINGO-4-YFP) or the Renilla luciferase (LINGO-1-Rluc, LINGO-2-RLuc, LINGO-3-RLuc or LINGO-4 RLuc), we observed a specific BRET signal generated by the formation of LINGO dimer/oligomers in living cells (Figure 6). The data first indicated that LINGO-1 form cis heterocomplexes with LINGO-2, LINGO-3 or LINGO-4 via direct protein-protein interactions. Then, the analysis of BRET saturation curves showed that the affinity between the protomers was very similar (comparable BRET $_{50}$ values) suggesting that LINGO-1 formed both homodimers and heterodimers with the same probability in heterologous system (Table 2). The difference in the BRETmax values observed between each homolog (Table 2) might probably reflects difference in the relative orientation of each BRET pairs. The specificity of the interactions was shown by the very low value of the non-specific energy transfer (best fit with a linear regression curve) measured in cells co-expressing LINGO constructs with an unrelated receptor, the vasopressine-2 receptor (V2R) (Fig S2). Altogether, these BRET analyses allowed us to demonstrate that LINGO-1 as well as its homologs are able to form homo- or heterocomplexes in living cells in the absence of exogenous ligands.

\section{LINGO-1 form heterocomplexes with its homologs in the mouse brain}


The formation of heteromers was then evaluated by co-immunoprecipitation analyses of the endogenous proteins expressed in the mouse cerebral cortex. We first validated the specificity of each LINGO antibody and their cross-reactivities with the homologs after transient expression of HA-tagged proteins in HEK-293 cells. As observed in Figure S3, we validated the specificity of the anti-LINGO-1 antibody that recognizes two prominent LINGO-1 species at 80 and $100 \mathrm{kDa}$. Western blotting with the LINGO-2 antibody allowed us to detect an immunoreactive species close to $100 \mathrm{kDa}$. LINGO-3 and LINGO-4 antibodies recognized specific immunoreactive species with two prominent labelling at $80 \mathrm{kDa}$ and $100 \mathrm{kDa}$ for LINGO-3, and one at $70 \mathrm{kDa}$ for LINGO-4. The difference in the size of LINGO homologs might reflect different glycosylation levels (27). We then validated that LINGO homologs could be detected and form complex in mammalian brain tissues by using immunoprecipitation assays. As shown in Figure 7, mouse cerebral cortex lysates were immunoprecipitated with anti-LINGO-1, then immunoblotted with anti-LINGO-1, antiLINGO-2, anti-LINGO-3 or anti-LINGO-4. In immunoprecipitates, LINGO-1 but also its homologs LINGO-2 as well as LINGO-3 and LINGO-4 were detected. Thus, endogenous LINGO homologs form heterocomplex in brain tissues.

\section{Discussion}

Recent data suggest that LINGO-1 is associated with different neurological and psychiatric diseases including, spinal cord injury, traumatic brain injury, multiple sclerosis, Parkinson's and Alzheimer's diseases, epilepsy and glaucoma $(9,10)$. The protein is recognized to transmit signals of several bound myelin associated inhibitors including Nogo-A (neurite outgrowth inhibitor), MAG (myelin-associated glycoprotein) and OMgp (oligodendrocyte myelin glycoprotein). Implicated in a ternary complex with the Nogo receptor (NgR1), which in the absence of a transmembrane domain, requires the activity of the p75 neurotrophin receptor (p75) or the TNF receptor orphan Y (TROY), LINGO-1 is also known to be associated with a high number of other co-receptors and co-factors. Consequently, the protein-protein interactions involving LINGO-1 result in the activation of many downstream signaling proteins (10). The complexity of the transduction of LINGO-mediated signals was further increased by the finding that LINGO-1 is able to form homotypic interactions shown by various approaches including results based on the determination of the crystal molecular structure of LINGO-1 ectodomain obtained(26), immunoprecipitation assays after cell solubilization $(14,32,33)$ and BRET analysis on intact cells (27). An even higher level of intricacy is also provided by the existence of the three LINGO-1 homologs (3). Now, our 
work suggests the possible existence of an additional level of control for the intracellular signaling triggered by the LINGO proteins supported by the transcription of the four LINGO homologs in several regions of the adult mouse brain, their co-expression at the cellular level and their ability to form homo- and heterodimers in those tissues.

In agreement with our results, the expression level of LINGO-1 transcripts and proteins was previously reported to be higher in the limbic system (namely including the hippocampus) and in cortical areas $(1,2,5)$. Except for LINGO-2 that appears to be expressed in a uniform manner and at a low level in all brain regions that we analyzed in the present work, the other homologs display expression levels depending on the brain area that is considered. Remarkably, if the four homologs can be detected in all regions, the region exhibiting the highest transcription varies from one homolog to the other one as attested by the highest expression level observed in the cerebral cortex, striatum and cerebellum for LINGO-1, LINGO-3 and LINGO-4, respectively. Although our co-immunolabeling experiments support the hypothesis that LINGO-1 is co-expressed with LINGO-2, LINGO-3 or LINGO-4, heterodimerization of LINGO proteins is likely not invariably the rule given the apparent differences of expression levels for each homolog within a single region. Although the hypothesis remains to be investigated, our ISH data also indicate that the expression profiles of LINGO homologs are different within a given region. This is namely true in the thalamus where LINGO-4 transcripts are more widely distributed than LINGO-1 or LINGO-3 suggesting again that the formation of heterodimers will not evenly occur throughout this brain region. Moreover, we show that like LINGO-1, its homologs LINGO-2, LINGO-3 and LINGO-4 are mostly expressed in neurons while they are only detected in a highly restricted population of Olig2-expressing oligodendroglial cells. In a consistent manner with its negative role on OPC differentiation and myelination, LINGO-1 is down-regulated during OPC maturation (32). Further work would be required to determine whether LINGO-1 homologs are similarly down-regulated in differentiating OPCs and thus participate in the control of early postnatal myelination.

The indisputable evidence that LINGO-1 proteins may interact with its homologs relies on BRET experiments on whole cells since the latter avoid aggregation of proteins due to the detergent present in the lysis buffer used in the co-immunoprecipitation approach. BRET saturation experiments have previously been successfully carried out in order to study the relative affinities of various pairs of interacting proteins $(34,35)$ and to estimate ratios between dimeric and monomeric fractions of the two BRET partners $(34,36)$. The difference 
in BRET $_{\max }$ values that we observed for all studied pairs likely reflects differences in the distance and/or orientation of each YFP and RLuc moieties between the proteins. The BRET 50 (the acceptor to donor ratio yielding $50 \%$ of the BRET $_{\text {max }}$ as derived from luminescence and fluorescence measurements) has been proposed to reflect the relative affinities of donor and acceptor fusion proteins $(34,35)$. We did not observe any significant variation in BRET $_{50}$ values between each pair suggesting that LINGO-1 has the same probability to form homoand heterodimers. Therefore, we propose that LINGO homolog heterodimerization may be involved in the regulation of LINGO-1 function. The physiological relevance of this hypothesis is supported by the co-immunoprecipitation experiments that we performed in the mouse cerebral cortex showing that LINGO-1 co-immunoprecipitates with LINGO-2, LINGO-3 and LINGO-4

Interestingly, $\mathrm{NgR}$ and p75 (or Troy) do not always co-exist with LINGO-1 (5) indicating that LINGO-1 may signal through additive mechanisms. The formation of oligomers might constitute one of these mechanisms. It is known that blocking of LINGO-1 oligomerization has beneficial effect on oligodendrocyte differentiation and the subsequent myelination of axons. Indeed, the monoclonal LINGO-1 antibody Li81 actually assessed in clinical trials for multiple sclerosis treatment mediates its positive effects on oligodendrocyte maturation by blocking LINGO-1 interface required for the oligomerization of the protein (14). In a consistent manner, our own work has demonstrated that LINGO-1 forms constitutive cis-dimers at the plasma membrane. The conformational state of these cis-dimers can be affected by low molecular weight compounds that we have identified (27). Therefore, modulation of the dimerization state by small molecules and/or antibodies may represent interesting pharmacological strategies to control LINGO-1 function. Furthermore, considering the sequence similarities that show a remarkable degree of conservation between species (Table 1), we may speculate that the formation of LINGO heterocomplexes must be also involved in critical processes in human.

It would be interesting to further characterize the protein domains involved in the formation of these heterocomplexes. LRRs constitute a consensus sequence characterized by 11 conserved aliphatic amino acids including leucine. LRR domain is thought to be involved in protein-protein interactions (37). The Ig domain is also found in many proteins including cell adhesion molecules and is responsible for homo- or heterophilic molecular interactions (38). In agreement with the possible involvement of the LRRIG domain in the formation of LINGO homolog heterocomplexes, LINGO-1 was reported to interact with other LRRIG 
proteins, such as the neurotrophin receptors or the Trk family members comprising three LRR and two Ig domains in their extracellular region (9). In addition, dozens of other proteins with LRR domains have been shown to play important roles in outgrowth of neuronal processes and synapse formation (9). Interestingly, LINGO-1 seems to participate in intercellular interactions through self-association in trans, a mechanism playing an important role in the inhibition of oligodendrocyte differentiation through the RhoA pathway (32). Although our BRET experiments demonstrate homo- and hetero-interactions of LINGO-1 in cis (on the same cell), we cannot exclude that interaction in trans can occur, as well and, participate in the regulation of neuronal functions.

\section{Acknowledgments}

We thank Fabienne Fasani (CBM, Orléans), Nadège Hervouet (CBM, Orléans), Chayma El Khamlichi (CBM, Orléans) and David Gosset (CBM, Orléans) for their technical advices. We also thank Arnaud Menuet (INEM, Orléans) and Céline Dubourg (INEM, Orléans) for helpful discussions.

\section{Author Contributions}

S. Morisset-Lopez, E. Traiffort designed the study; A. Guillemain, Y. Laouarem, L. Cobret, D. Stefok, W. Chen, S. Bloch, A. Zahaf and L. Blot performed experiments; A. Guillemain, Y. Laouarem, L. Cobret, D. Stefok and W. Chen were involved in data analysis; F. Reverchon was involved in sample acquisition; T. Normand provided analytic tools to perform experiments; S. Morisset-Lopez, E. Traiffort, C. Grillon and M. Decoville were involved in funding acquisition; S. Morisset-Lopez and E. Traiffort wrote the paper.

\section{Supporting information}

Additional supporting information may be found online in the Supporting Information section.

\section{Notes}

The authors declare no competing financial interests. 


\section{References}

1. Carim-Todd, L., Escarceller, M., Estivill, X., and Sumoy, L. (2003) LRRN6A/LERN1 (leucine-rich repeat neuronal protein 1 ), a novel gene with enriched expression in limbic system and neocortex. Eur J Neurosci 18, 3167-3182

2. Homma, S., Shimada, T., Hikake, T., and Yaginuma, H. (2009) Expression pattern of LRR and Ig domain-containing protein (LRRIG protein) in the early mouse embryo. Gene Expr Patterns $\mathbf{9}$, $1-26$

3. Chen, Y., Aulia, S., Li, L., and Tang, B. L. (2006) AMIGO and friends: an emerging family of brain-enriched, neuronal growth modulating, type I transmembrane proteins with leucinerich repeats (LRR) and cell adhesion molecule motifs. Brain Res Rev 51, 265-274

4. Barrette, B., Vallieres, N., Dube, M., and Lacroix, S. (2007) Expression profile of receptors for myelin-associated inhibitors of axonal regeneration in the intact and injured mouse central nervous system. Mol Cell Neurosci 34, 519-538

5. Llorens, F., Gil, V., Iraola, S., Carim-Todd, L., Marti, E., Estivill, X., Soriano, E., del Rio, J. A., and Sumoy, L. (2008) Developmental analysis of Lingo-1/Lern1 protein expression in the mouse brain: interaction of its intracellular domain with Myt1l. Dev Neurobiol 68, 521-541

6. Mi, S., Lee, X., Shao, Z., Thill, G., Ji, B., Relton, J., Levesque, M., Allaire, N., Perrin, S., Sands, B., Crowell, T., Cate, R. L., McCoy, J. M., and Pepinsky, R. B. (2004) LINGO-1 is a component of the Nogo-66 receptor/p75 signaling complex. Nat Neurosci 7, 221-228

7. Okafuji, T., and Tanaka, H. (2005) Expression pattern of LINGO-1 in the developing nervous system of the chick embryo. Gene Expr Patterns 6, 57-62

8. Haines, B. P., and Rigby, P. W. (2008) Expression of the Lingo/LERN gene family during mouse embryogenesis. Gene Expr Patterns 8, 79-86

9. Andrews, J. L., and Fernandez-Enright, F. (2015) A decade from discovery to therapy: Lingo-1, the dark horse in neurological and psychiatric disorders. Neurosci Biobehav Rev 56, 97-114

10. Llorens, F., Gil, V., and del Rio, J. A. (2011) Emerging functions of myelin-associated proteins during development, neuronal plasticity, and neurodegeneration. FASEB J 25, 463-475

11. Mi, S., Pepinsky, R. B., and Cadavid, D. (2013) Blocking LINGO-1 as a therapy to promote CNS repair: from concept to the clinic. CNS drugs 27, 493-503

12. Petrillo, J., Balcer, L., Galetta, S., Chai, Y., Xu, L., and Cadavid, D. (2019) Initial Impairment and Recovery of Vision-Related Functioning in Participants With Acute Optic Neuritis From the RENEW Trial of Opicinumab. J Neuroophthalmol 39, 153-160

13. Aktas, O., Albrecht, P., and Hartung, H. P. (2016) Optic neuritis as a phase 2 paradigm for neuroprotection therapies of multiple sclerosis: update on current trials and perspectives. Curr Opin Neurol 29, 199-204

14. Pepinsky, R. B., Arndt, J. W., Quan, C., Gao, Y., Quintero-Monzon, O., Lee, X., and Mi, S. (2014) Structure of the LINGO-1-anti-LINGO-1 Li81 antibody complex provides insights into the biology of LINGO-1 and the mechanism of action of the antibody therapy. J Pharmacol Exp Ther 350, 110-123

15. Shao, Z., Lee, X., Huang, G., Sheng, G., Henderson, C. E., Louvard, D., Sohn, J., Pepinsky, B., and $\mathrm{Mi}, \mathrm{S}$. (2017) LINGO-1 Regulates Oligodendrocyte Differentiation through the Cytoplasmic Gelsolin Signaling Pathway. J Neurosci 37, 3127-3137

16. Cadavid, D., Mellion, M., Hupperts, R., Edwards, K. R., Calabresi, P. A., Drulovic, J., Giovannoni, G., Hartung, H. P., Arnold, D. L., Fisher, E., Rudick, R., Mi, S., Chai, Y., Li, J., Zhang, Y., Cheng, W., Xu, L., Zhu, B., Green, S. M., Chang, I., Deykin, A., Sheikh, S. I., and investigators, S. s. (2019) Safety and efficacy of opicinumab in patients with relapsing multiple sclerosis (SYNERGY): a randomised, placebo-controlled, phase 2 trial. Lancet Neurol

17. Park, J. B., Yiu, G., Kaneko, S., Wang, J., Chang, J., He, X. L., Garcia, K. C., and He, Z. (2005) A TNF receptor family member, TROY, is a coreceptor with Nogo receptor in mediating the inhibitory activity of myelin inhibitors. Neuron 45, 345-351 
18. Shao, Z., Browning, J. L., Lee, X., Scott, M. L., Shulga-Morskaya, S., Allaire, N., Thill, G., Levesque, M., Sah, D., McCoy, J. M., Murray, B., Jung, V., Pepinsky, R. B., and Mi, S. (2005) TAJ/TROY, an orphan TNF receptor family member, binds Nogo-66 receptor 1 and regulates axonal regeneration. Neuron 45, 353-359

19. Yamashita, T., Fujitani, M., Yamagishi, S., Hata, K., and Mimura, F. (2005) Multiple signals regulate axon regeneration through the Nogo receptor complex. Mol Neurobiol 32, 105-111

20. Inoue, H., Lin, L., Lee, X., Shao, Z., Mendes, S., Snodgrass-Belt, P., Sweigard, H., Engber, T., Pepinsky, B., Yang, L., Beal, M. F., Mi, S., and Isacson, O. (2007) Inhibition of the leucine-rich repeat protein LINGO-1 enhances survival, structure, and function of dopaminergic neurons in Parkinson's disease models. Proc Natl Acad Sci U S A 104, 14430-14435

21. Lee, X., Shao, Z., Sheng, G., Pepinsky, B., and Mi, S. (2014) LINGO-1 regulates oligodendrocyte differentiation by inhibiting ErbB2 translocation and activation in lipid rafts. Mol Cell Neurosci 60, 36-42

22. Mandai, K., Guo, T., St Hillaire, C., Meabon, J. S., Kanning, K. C., Bothwell, M., and Ginty, D. D. (2009) LIG family receptor tyrosine kinase-associated proteins modulate growth factor signals during neural development. Neuron 63, 614-627

23. Meabon, J. S., de Laat, R., leguchi, K., Serbzhinsky, D., Hudson, M. P., Huber, B. R., Wiley, J. C., and Bothwell, M. (2016) Intracellular LINGO-1 negatively regulates Trk neurotrophin receptor signaling. Mol Cell Neurosci 70, 1-10

24. Zhang, Z., Xu, X., Zhang, Y., Zhou, J., Yu, Z., and He, C. (2009) LINGO-1 interacts with WNK1 to regulate nogo-induced inhibition of neurite extension. J Biol Chem 284, 15717-15728

25. Zhang, Z., Xu, X., Xiang, Z., Yu, Z., Feng, J., and He, C. (2013) LINGO-1 receptor promotes neuronal apoptosis by inhibiting WNK3 kinase activity. J Biol Chem 288, 12152-12160

26. Mosyak, L., Wood, A., Dwyer, B., Buddha, M., Johnson, M., Aulabaugh, A., Zhong, X., Presman, E., Benard, S., Kelleher, K., Wilhelm, J., Stahl, M. L., Kriz, R., Gao, Y., Cao, Z., Ling, H. P., Pangalos, M. N., Walsh, F. S., and Somers, W. S. (2006) The structure of the Lingo-1 ectodomain, a module implicated in central nervous system repair inhibition. $J$ Biol Chem 281, 36378-36390

27. Cobret, L., De Tauzia, M. L., Ferent, J., Traiffort, E., Henaoui, I., Godin, F., Kellenberger, E., Rognan, D., Pantel, J., Benedetti, H., and Morisset-Lopez, S. (2015) Targeting the cisdimerization of LINGO-1 with low MW compounds affects its downstream signalling. $\mathrm{Br} \mathrm{J}$ Pharmacol 172, 841-856

28. Traiffort, E., Charytoniuk, D., Watroba, L., Faure, H., Sales, N., and Ruat, M. (1999) Discrete localizations of hedgehog signalling components in the developing and adult rat nervous system. Eur J Neurosci 11, 3199-3214

29. Achour, L., Kamal, M., Jockers, R., and Marullo, S. (2011) Using quantitative BRET to assess G protein-coupled receptor homo- and heterodimerization. Methods Mol Biol 756, 183-200

30. El Khamlichi, C., Reverchon-Assadi, F., Hervouet-Coste, N., Blot, L., Reiter, E., and MorissetLopez, S. (2019) Bioluminescence Resonance Energy Transfer as a Method to Study ProteinProtein Interactions: Application to G Protein Coupled Receptor Biology. Molecules 24

31. James, J. R., Oliveira, M. I., Carmo, A. M., Iaboni, A., and Davis, S. J. (2006) A rigorous experimental framework for detecting protein oligomerization using bioluminescence resonance energy transfer. Nat Methods 3, 1001-1006

32. Jepson, S., Vought, B., Gross, C. H., Gan, L., Austen, D., Frantz, J. D., Zwahlen, J., Lowe, D., Markland, W., and Krauss, R. (2012) LINGO-1, a Transmembrane Signaling Protein, Inhibits Oligodendrocyte Differentiation and Myelination through Intercellular Self-interactions. J Biol Chem 287, 22184-22195

33. Stein, T., and Walmsley, A. R. (2012) The leucine-rich repeats of LINGO-1 are not required for self-interaction or interaction with the amyloid precursor protein. Neurosci Lett 509, 9-12

34. Mercier, J. F., Salahpour, A., Angers, S., Breit, A., and Bouvier, M. (2002) Quantitative assessment of beta 1- and beta 2-adrenergic receptor homo- and heterodimerization by bioluminescence resonance energy transfer. J Biol Chem 277, 44925-44931 
35. Wang, D., Sun, X., Bohn, L. M., and Sadee, W. (2005) Opioid receptor homo- and heterodimerization in living cells by quantitative bioluminescence resonance energy transfer. Mol Pharmacol 67, 2173-2184

36. Couturier, C., and Jockers, R. (2003) Activation of the leptin receptor by a ligand-induced conformational change of constitutive receptor dimers. J Biol Chem 278, 26604-26611

37. Kobe, B., and Kajava, A. V. (2001) The leucine-rich repeat as a protein recognition motif. Curr Opin Struct Biol 11, 725-732

38. Maness, P. F., and Schachner, M. (2007) Neural recognition molecules of the immunoglobulin superfamily: signaling transducers of axon guidance and neuronal migration. Nat Neurosci 10, $19-26$

\section{Figure legends}

Figure 1. Analysis of LINGO expression in various brain regions by quantitative PCR analysis. The relative expressions of transcripts encoding LINGO-1, LINGO-2, LINGO-3 and LINGO-4 were determined in the indicated regions from the adult mouse brain. Data are the mean \pm SEM from $n=6$ animals.

Figure 2. Wide transcription of LINGO homologs in the adult mouse brain. In situ hybridization of LINGO-1, LINGO-2, LINGO-3 and LINGO-4 in the cerebral cortex (A), striatum (B), hippocampus (C), thalamus (D) and cerebellum (E). Abbreviations: CA, Ammon's horn; cc, corpus callosum; DCN, deep cerebellar nuclei; GL, granular layer; Int, internal capsule; PL, Purkinke cell layer; St, striatum; Th, thalamus. Scale bars: $100 \mu \mathrm{m}$ (A), $200 \mu \mathrm{m}(\mathrm{B}-\mathrm{E})$.

Figure 3. Determination of the phenotype of cells expressing LINGO homologs. (A) Double in situ hybridization of the LINGO-1 riboprobe and immunostainings using NeuN or Olig2 antibodies. (B, C) Quantification of $\mathrm{LINGO}-1^{+}, \mathrm{LINGO}-2^{+}, \mathrm{LINGO}-3^{+}$and $\mathrm{LINGO}-4^{+}$ cells co-expressing NeuN or Olig2.

Figure 4: Expression and colocalization of LINGO homologs in the adult mouse brain. (A) Visualization of LINGO $-3^{+}$and $\mathrm{MAP}^{+}$neurons in the cerebral cortex from an adult mouse. Nuclei are stained with DAPI (blue). (B-D) Co-immunolabelling of LINGO-1 with its homologs observed in the cerebral cortex for LINGO-2 and LINGO-3, or in the cerebellum for LINGO-4. In each panel, the area delineated with a white frame is magnified in the insets showing split fluorescent channels. 
Figure 5: Homotypic interactions of LINGO-1 in HEK 293 cells. (A, B) Immunoprecipitation experiments were performed to evaluate homo-oligomerization (A) or hetero-oligomerization (B). The HA-tagged LINGO-1 was expressed transiently in HEK 293 cells in the presence or absence of YFP-tagged-LINGO-1 or homologs. 1/25 of each lysate was used as an input control (lysates). Cell lysates were immunoprecipitated with HA antibodies (HA-IP) and then analysed by $10 \%$ or $8 \%$ SDS/PAGE and immunobloted for YFP and HA-tagged constructs.

Figure 6. Saturation BRET experiments showing the formation of cis-homo and heterooligomers of LINGO homologs. Homotypic (A) and heterotypic (B) interaction between LINGO homologs was studied in adherent HEK 293 cells transiently transfected with a constant DNA amount for RLuc construct ( $25 \mathrm{ng}$ ) and increasing amounts (from $0 \mathrm{ng}$ to 2000 ng) for YFP constructs. The difference between the BRET ratio obtained in cells coexpressing LINGO-Rluc and LINGO-YFP (BRET ratio varied from 0.85 to 2.45 ) and the BRET ratio of cells expressing only the LINGO-RLuc fusion protein alone (BRET ratio= 0.85) was defined as the net BRET. Results were expressed in milliBRET units (mBU, with 1 mBU corresponding to the net BRET values multiplied by 1000). The BRET signal observed between LINGO fusion proteins increases as a hyperbolic curve reaching an asymptote underlying the specificity of the signal. BRET $_{50}$ values and BRET $_{\max }$ values presented in Table 2 were deduced from these data analysis using a nonlinear regression equation applied to a single binding site model. Data represent the mean of three independent experiments read in triplicates.

Figure 7. Detection of hetero-oligomers of LINGO homologs in mouse brain. (A) LINGO proteins were detected in total cell lysates from all brain regions. (B) After immunoprecipitation of LINGO-1 from cerebral cortex lysates with anti-LINGO-1 antibody, its homologs LINGO-2, LINGO-3 and LINGO-4 could be detected in the immunoprecipitate. Western blots are representative of two experiments. 
Table 1 Percentage of amino acid identity between human and mouse LINGO family members $^{\mathrm{a}}$

\begin{tabular}{ccccc}
\hline & $\begin{array}{c}\text { Human } \\
\text { LINGO-1 }\end{array}$ & $\begin{array}{c}\text { Human } \\
\text { LINGO-2 }\end{array}$ & $\begin{array}{c}\text { Human } \\
\text { LINGO-3 }\end{array}$ & $\begin{array}{c}\text { Human } \\
\text { LINGO-4 }\end{array}$ \\
\hline Human LINGO-1 & 100 & 61 & 55 & 44 \\
Human LINGO-2 & 61 & 100 & 58 & 44 \\
Human LINGO-3 & 55 & 58 & 100 & 45 \\
Human LINGO-4 & 44 & 44 & 45 & 100 \\
Mouse LINGO-1 & 99 & & & \\
Mouse LINGO-2 & & 97 & & \\
Mouse LINGO-3 & & & & \\
Mouse LINGO-4 & & & & \\
\hline
\end{tabular}

${ }^{a}$ For mouse LINGO, only comparisons to human orthologs are shown

Table 2. Parameters from BRET saturation curves

\begin{tabular}{cccc}
\hline Pair Transfected & BRET max & BRET 50 \\
\hline HOMOMERS & LINGO-1-RLuc + LINGO-1-YFP & $1690 \pm 83$ & $2.2 \pm 0.4$ \\
& LINGO-2-RLuc + LINGO-2-YFP & $309 \pm 20$ & $1.4 \pm 0.4$ \\
& LINGO-3-RLuc + LINGO-3-YFP & $537 \pm 24$ & $1.5 \pm 0.3$ \\
& LINGO-4-RLuc + LINGO-4-YFP & $1455 \pm 68$ & $1.5 \pm 0.2$ \\
HETEROMERS & & & \\
& & & $1 \pm 0.1$ \\
& LINGO-1-RLuc + LINGO-2-YFP & $156 \pm 6$ & $2.7 \pm 0.2$ \\
& LINGO-1-RLuc + LINGO-3-YFP & $771 \pm 15$ & $1.7 \pm 0.2$ \\
& LINGO-1-RLuc + LINGO-4-YFP & $807 \pm 34$ & \\
\hline
\end{tabular}

The $\mathrm{BRET}_{\max }$ is the maximal BRET ratio obtained for a given pair. The $\mathrm{BRET}_{50}$ represents the acceptor/donor ratio required to reach half-maximal BRET values. Results are the mean \pm SEM of three experiments performed in triplicates. 
LINGO-1

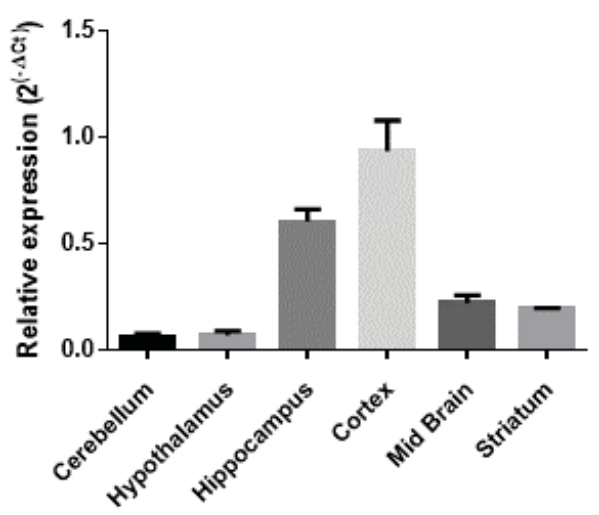

LINGO-3

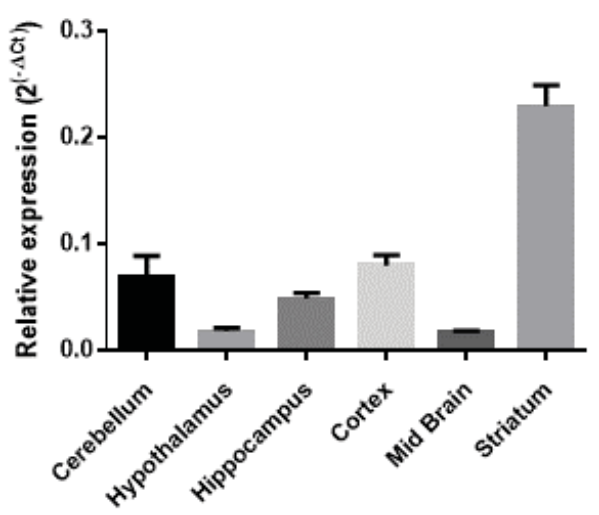

LINGO-2

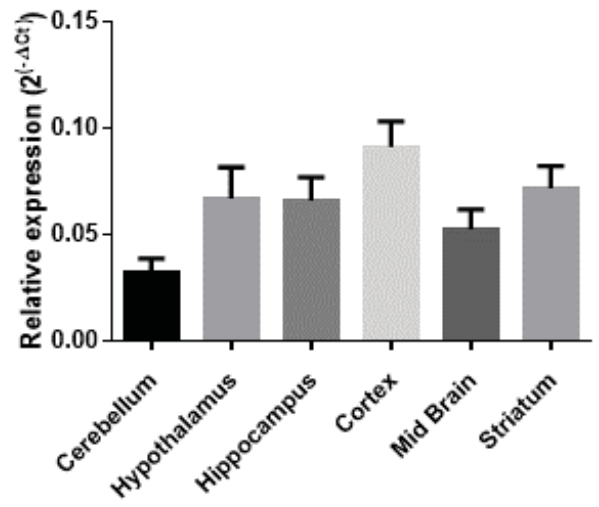

LINGO-4

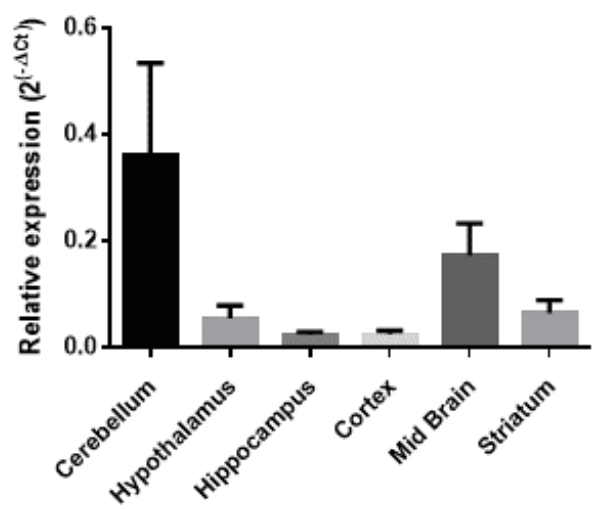


Figure 2

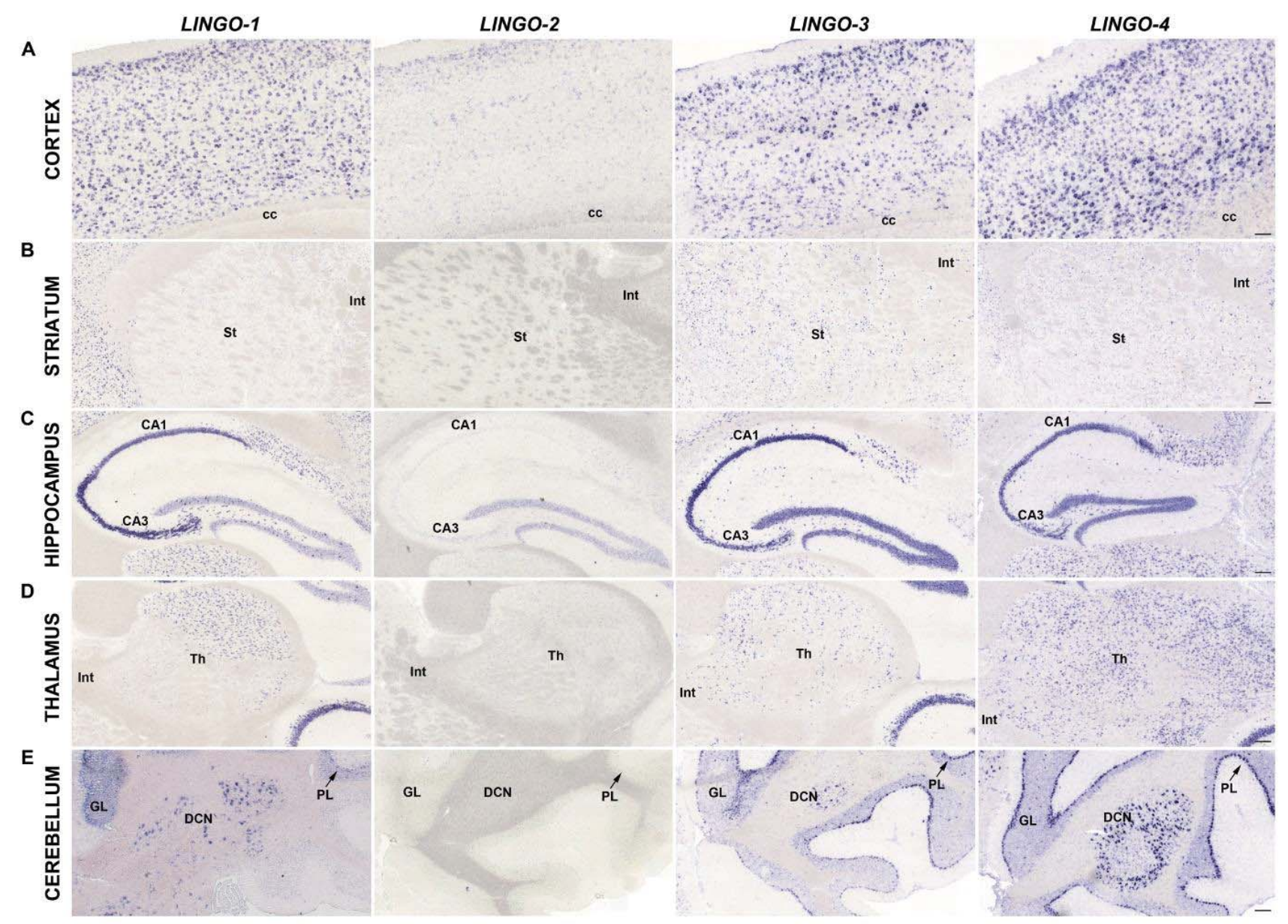




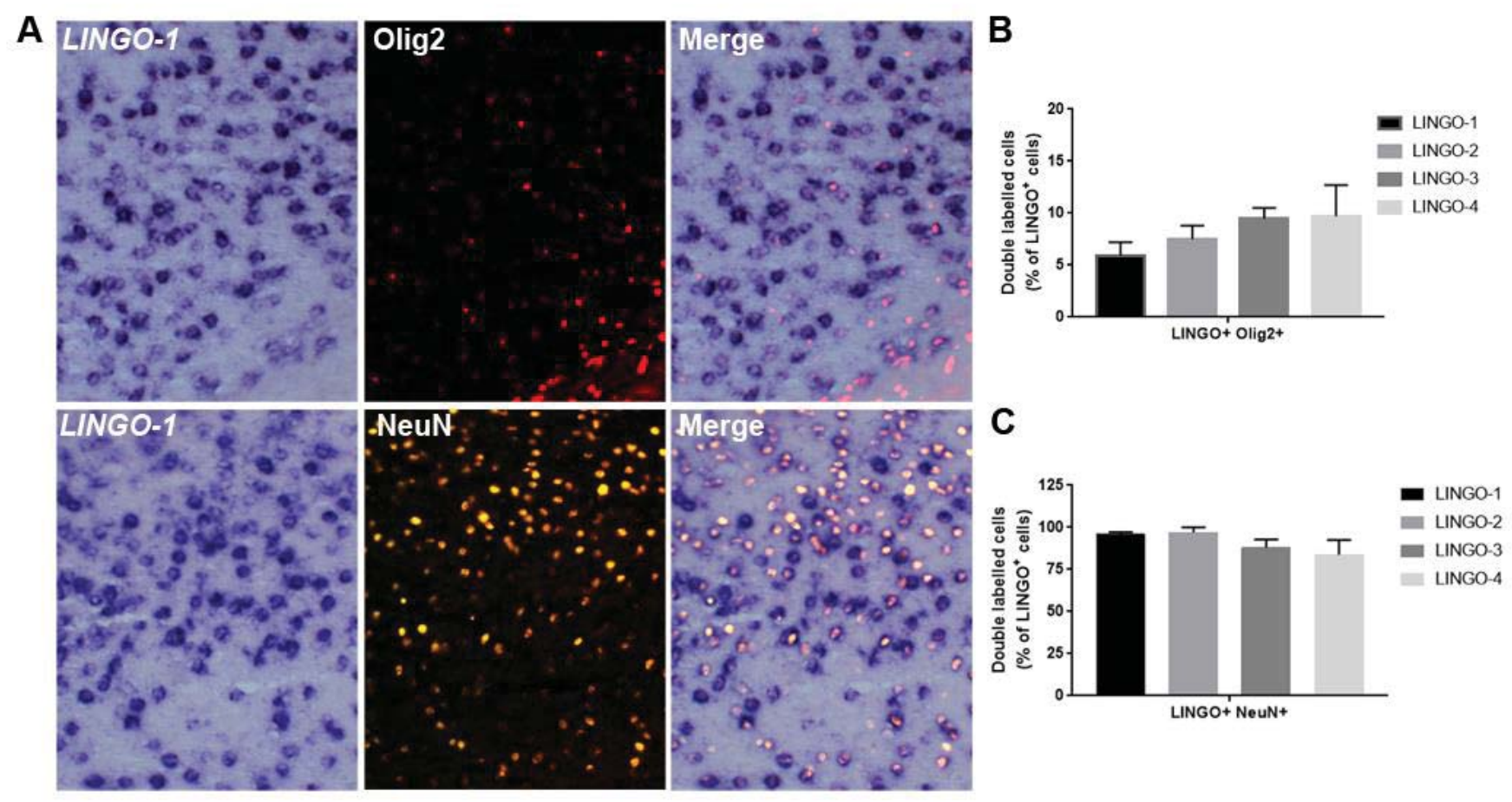


Figure 4

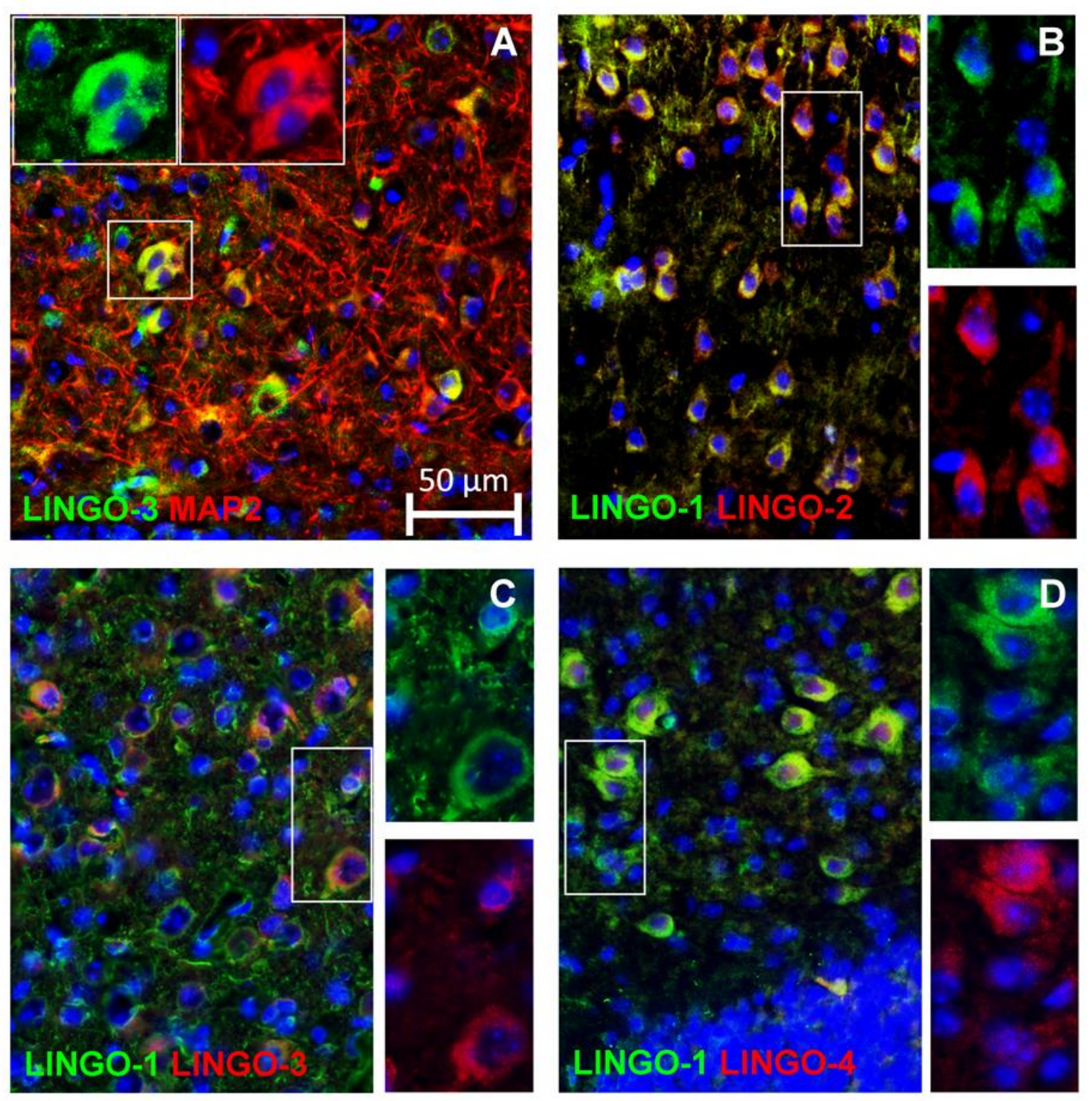


A

LINGO-2/LINGO-2

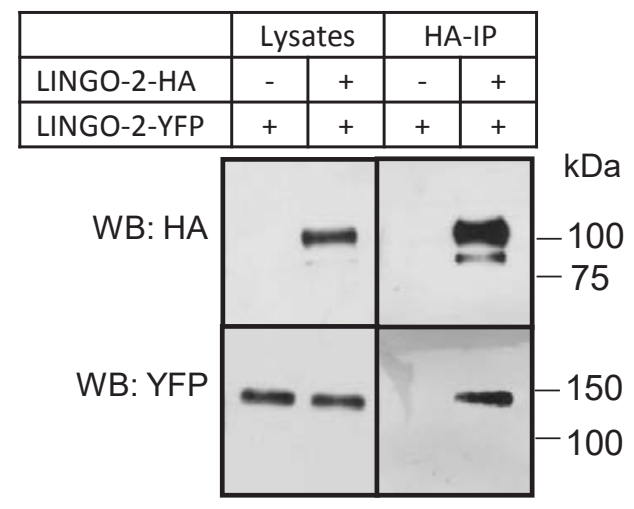

B

LINGO-1/LINGO-2

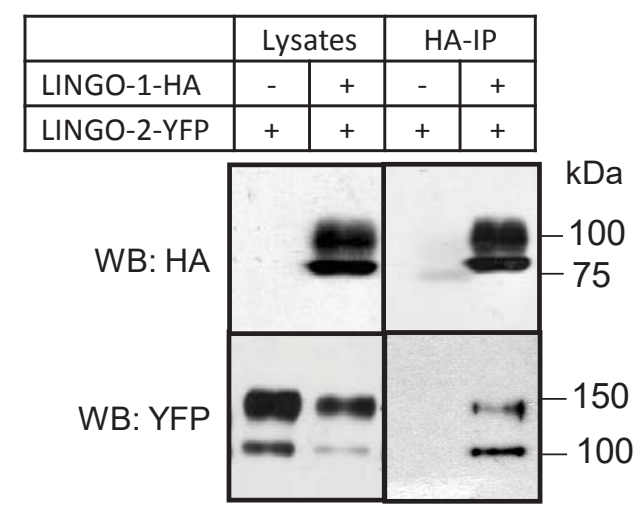

LINGO-3/LINGO-3

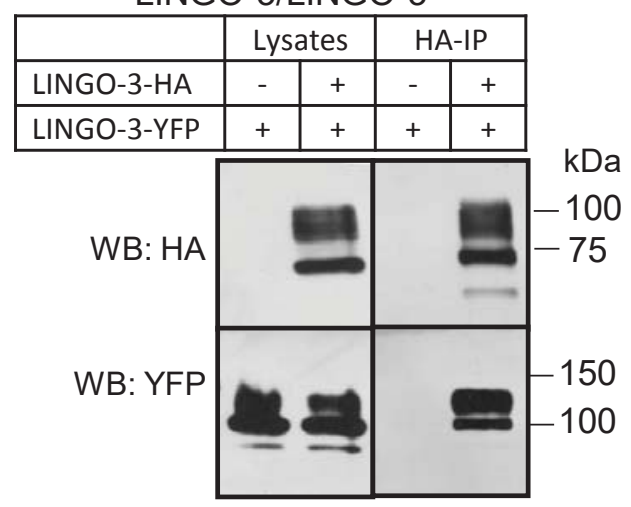

LINGO-1/LINGO-3

\begin{tabular}{|l|c|c|c|c|}
\hline & \multicolumn{2}{|c|}{ Lysates } & \multicolumn{2}{|c|}{ HA-IP } \\
\hline LINGO-1-HA & - & + & - & + \\
\hline LINGO-3-YFP & + & + & + & + \\
\hline
\end{tabular}

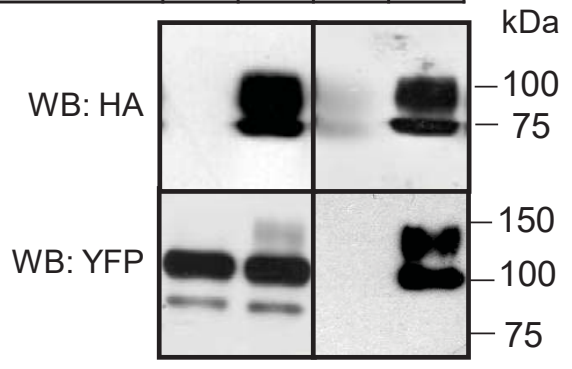

LINGO-4/LINGO-4

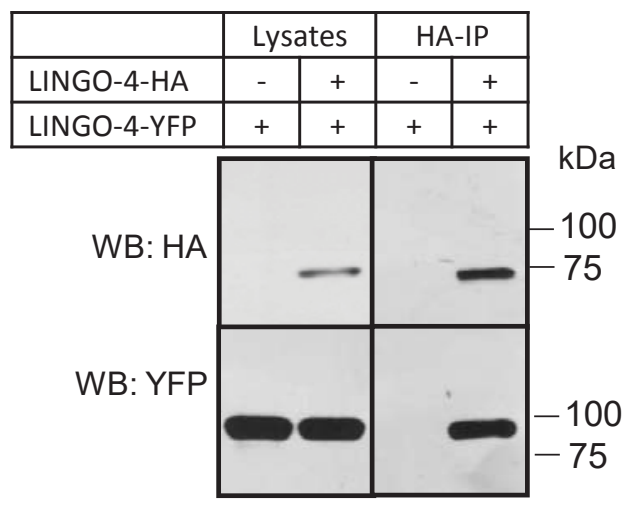

LINGO-1/LINGO-4

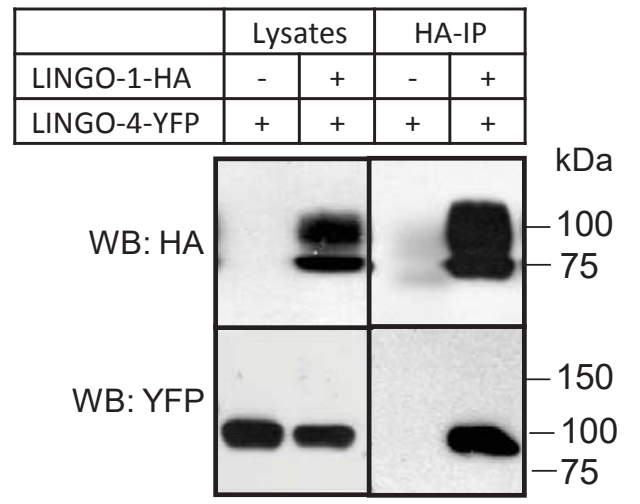


A

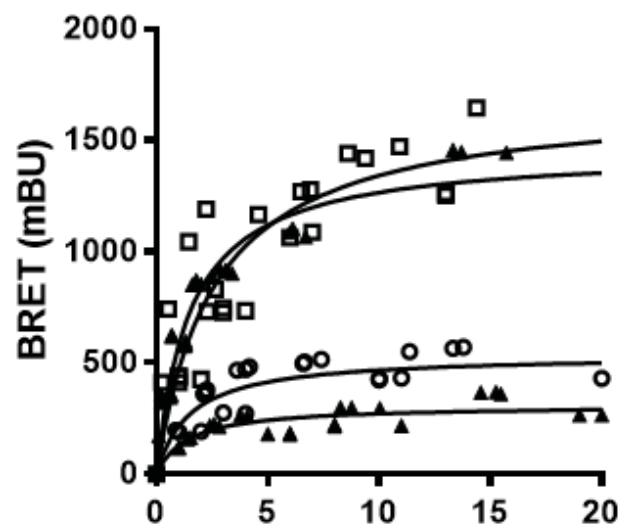

Fluorescence/Luminescence

a LINGO-1-RLuc + LINGO-1-YFP

- LINGO-2-RLuc + LINGO-2-YFP

- LINGO-3-RLuc + LINGO-3-YFP

- LINGO-4-RLuc + LINGO-4-YFP
B

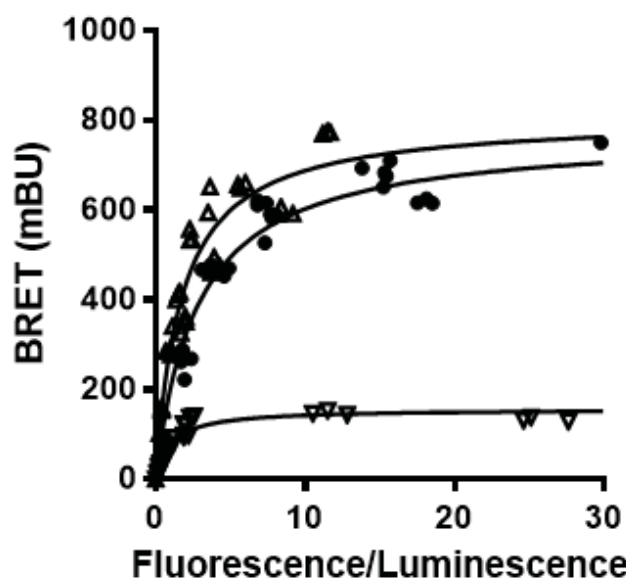

$\boldsymbol{\nabla}$ LINGO-1-Rluc - LINGO-2-YFP

- LINGO-1-Rluc - LINGO-3-YFP

$\Delta \quad$ LINGO-1-Rluc - LINGO-4-YFP 

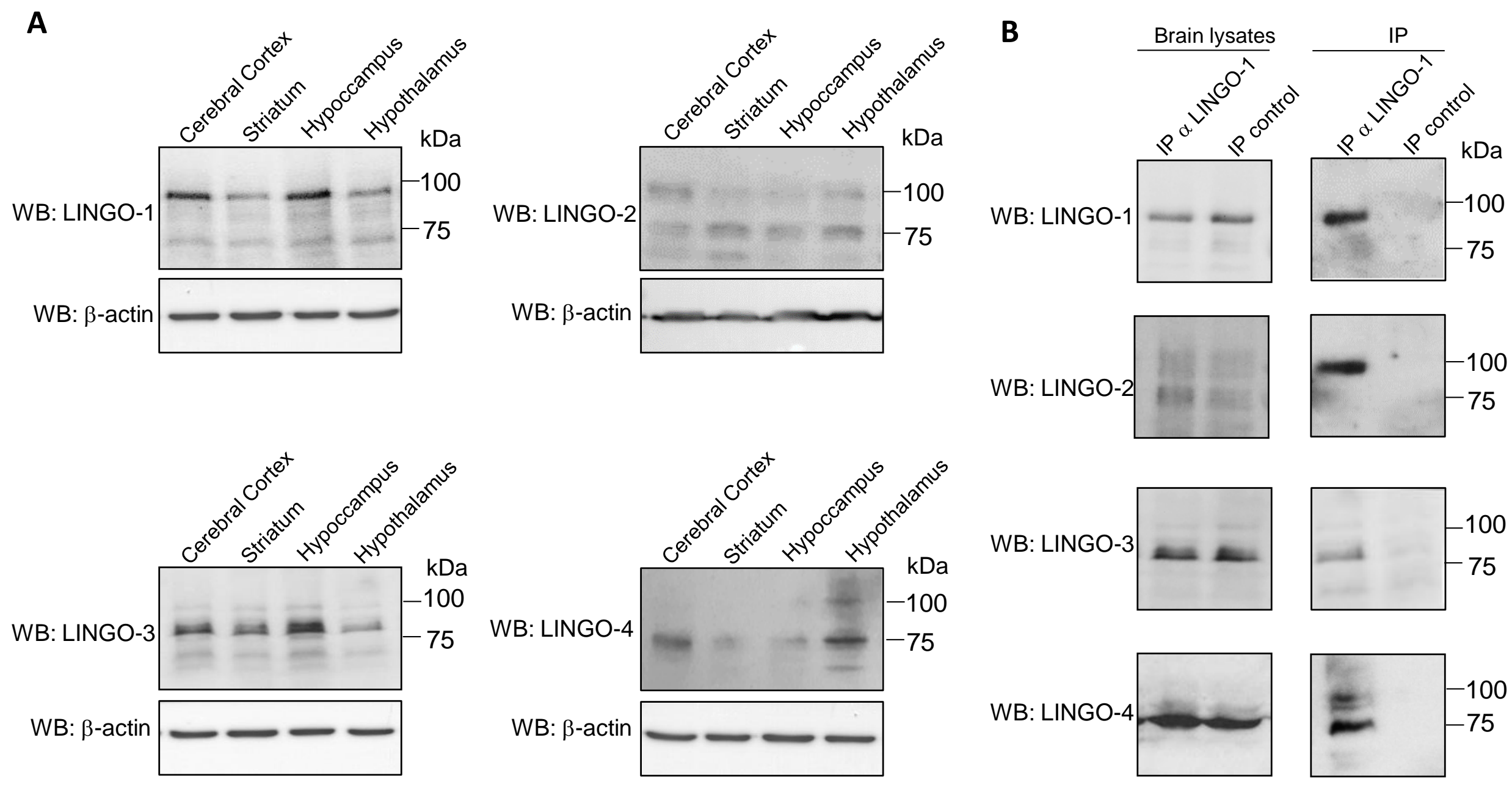
Supplemental information

\section{LINGO FAMILY RECEPTORS ARE DIFFERENTIALLY EXPRESSED IN THE MOUSE BRAIN AND FORM NATIVE MULTIMERIC COMPLEXES}

Anthony Guillemain ${ }^{1}$, Yousra Laouarem ${ }^{2}$, Laetitia Cobret ${ }^{1}$, Dora Stefok ${ }^{1}$, Wanyin Chen $^{1}$, Solal Bloch ${ }^{2}$, Amina Zahaf ${ }^{2}$, Lauren Blot ${ }^{1}$, Flora Reverchon ${ }^{1}$, Thierry Normand ${ }^{1}$, Martine Decoville $^{1}$, Catherine Grillon ${ }^{1}$, Elisabeth Traiffort ${ }^{2}$ and Séverine Morisset ${ }^{1}$ 


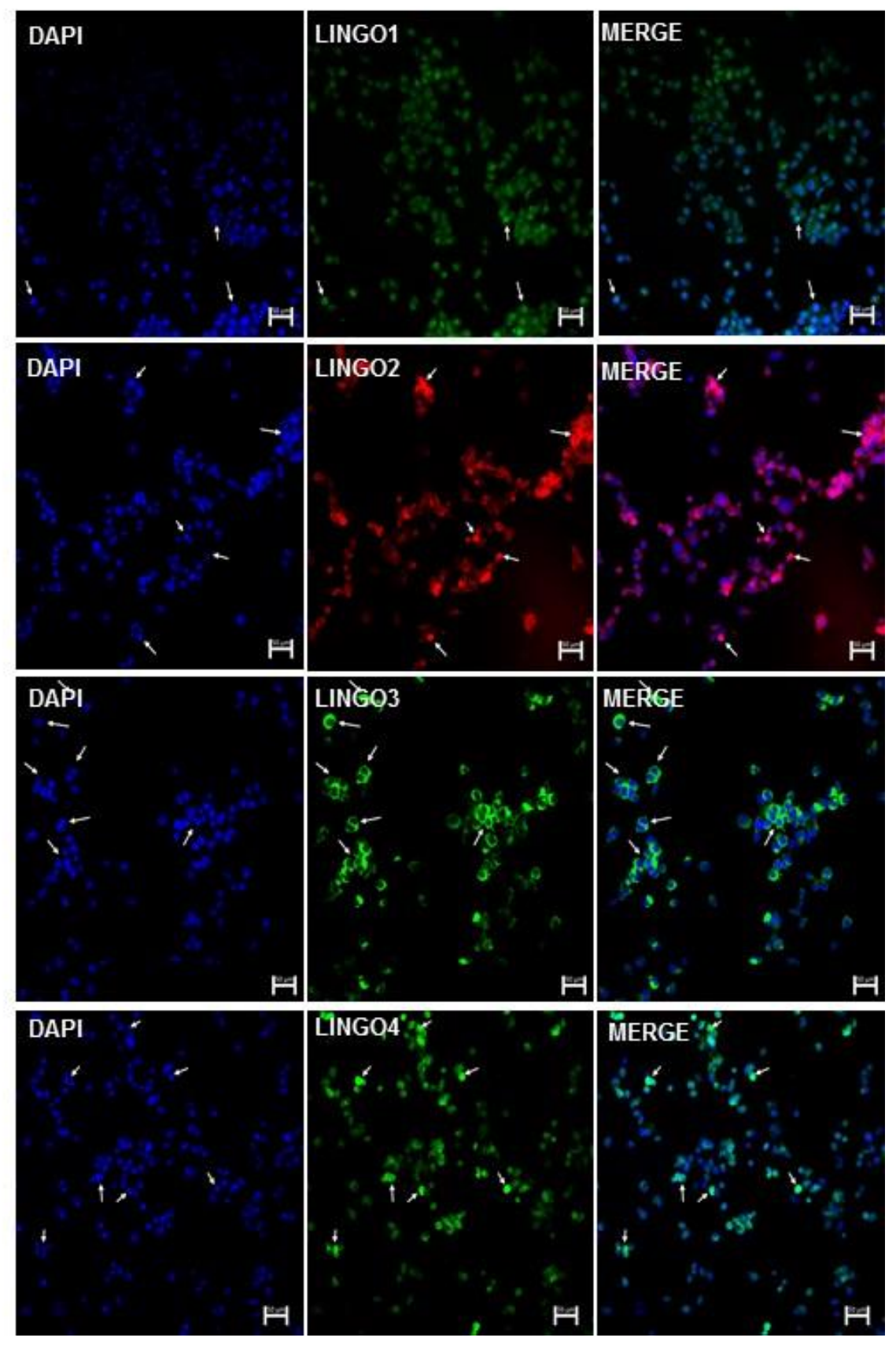

Figure S1: Expression of LINGO homologs in NG108-15 cell line. After fixation with PFA $4 \%$, endogenous expression of each LINGO homologs was evaluated by immunocytochemistry For this purpose, the cells, fixed on glass cover slides, were washed three times in TBS and incubated with 0,2M Urea. After a saturation step (1\% BSA, 5\% goat serum, $0,3 \%$ Triton 100X, TBS for one hour), cells were incubated with primary antibodies: anti-LINGO-1 (Abcam), anti-LINGO-2 (R\&D Systems), anti-LINGO-3 (Abcam) or antiLINGO-4 (Abgent) overnight at $4^{\circ} \mathrm{C}$ in wet chamber. Next day, after washing steps, cells were incubated with secondary antibody for 1 hour at room temperature. Then, they were treated with Bisbenzimide during $10 \mathrm{~min}$ for the visualization of nucleus and washed with TBS. Mounting was performed with Fluoromont-G solution. Scale bar: $50 \mu \mathrm{m}$. 

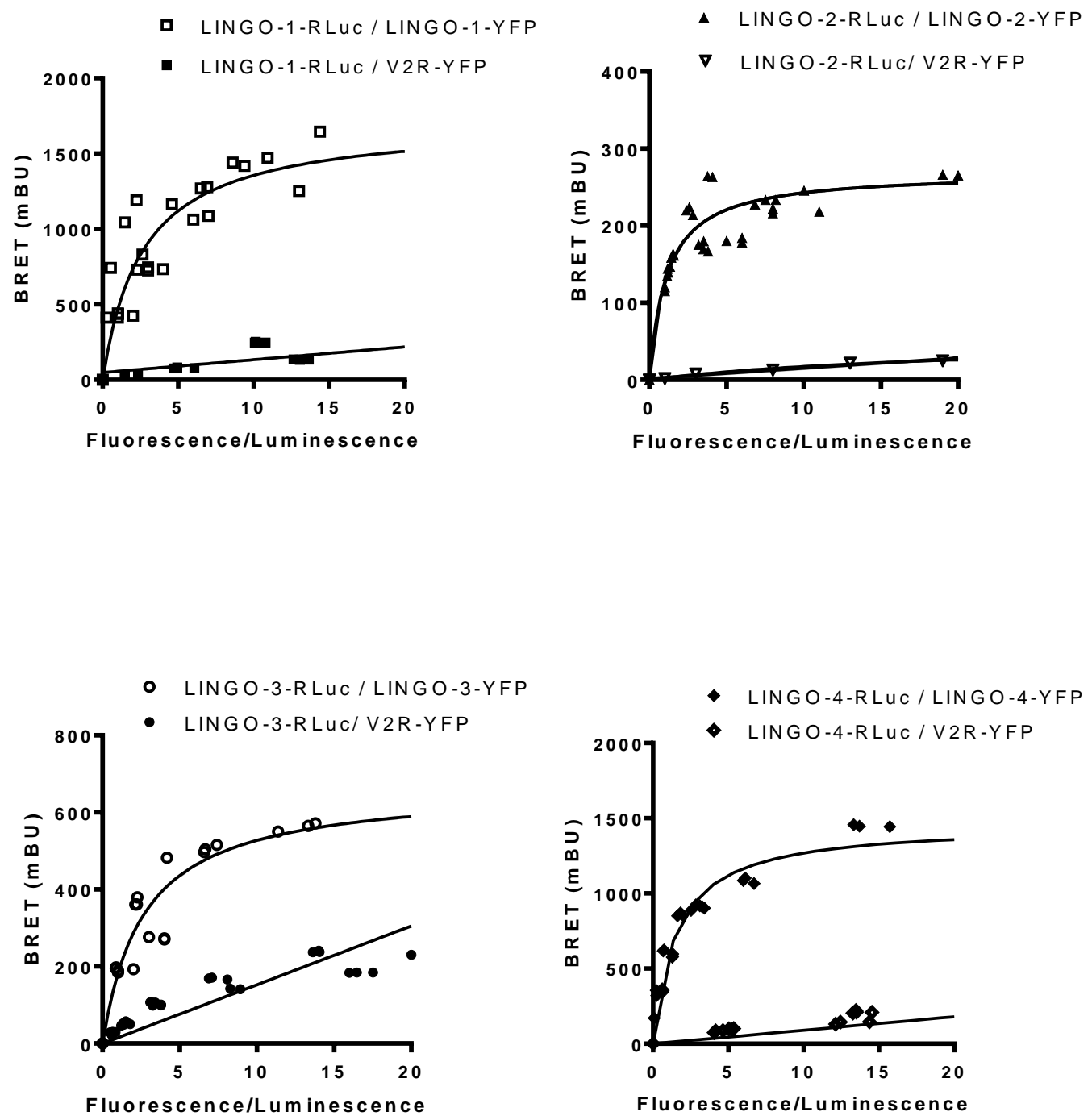

Figure S2. Specificity of BRET signals formed by LINGO complexes. HEK-293 cells were transfected transiently with a constant DNA amount (25ng) of BRET donor, LINGO-1 (or LINGO-2, LINGO-3, LINGO-4) fused to Rluc and increasing amounts (from $0 \mathrm{ng}$ to 2 $\mu \mathrm{g}$ ) of acceptors, LINGO-1 (or LINGO2, LINGO-3, LINGO-4) or vasopressin 2 receptor (V2R) fused to YFP. The BRET signal observed between LINGO homologs increases as a hyperbolic curve reaching an asymptote underlying the specificity of the signal. When LINGO-Rluc was co-expressed with the V2R, a protein belonging to the unrelated family of $\mathrm{G}$ protein-coupled receptors, the BRET signal is much lower and increases linearly indicating the occurrence of non-specific and random interactions. Data represent the mean of two or three independent experiments read in triplicates. 


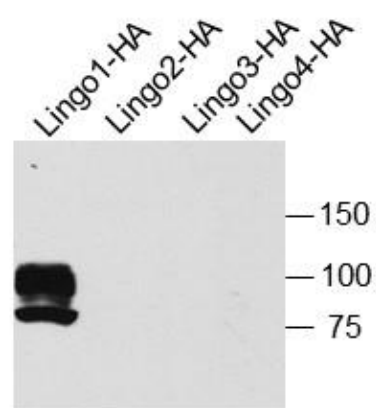

WB: anti-LINGO-1

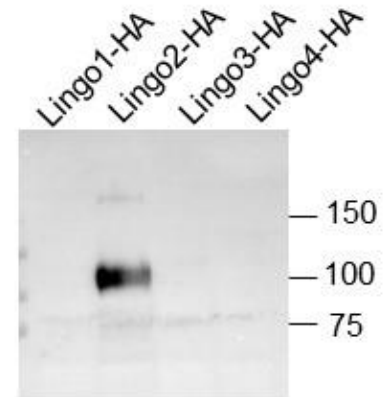

WB: anti-LINGO-2

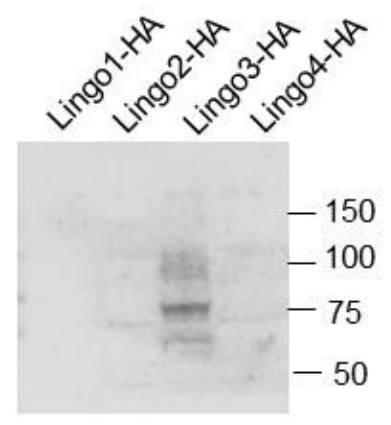

WB: anti-LINGO-3

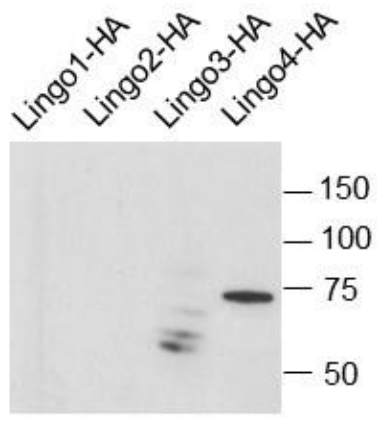

WB: anti-LINGO-4

Figure S3: Validation of the specificity of LINGO antibodies and absence of crossreactivity. HEK-293 cells were transfected transiently with LINGO homologs tagged with $\mathrm{HA}$ at the C-terminal part of the protein. For assessment of protein expression by Western blot analysis, lysates from HEK-293 transfected cells were separated by electrophoresis on SDS/PAGE and transferred on polyvinylidene fluoride (PVDF) membranes. Blots containing LINGO proteins were probed with LINGO antibodies: anti-LINGO-1 (R\&D Systems AF3086, goat 1:500); anti-LINGO-2 (R\&D Systems AF3679, goat 1:40), anti-LINGO-3 (Abcepta AP16177b, rabbit 1:1000) and anti-LINGO-4 (Santa-Cruz Biotechnology sc164871, goat 1:500). 